\title{
LA REFORMA DE LA EDUCACIÓN SUPERIOR EN MÉXICO, ENTRE LA ESPADA Y LA PARED. LA MIRADA DE LOS UNIVERSITARIOS
}

THE HIGHER EDUCATION REFORM IN MEXICO, BETWEEN A ROCK AND A HARD PLACE. THE LOOK OF THE UNIVERSITARIES

\author{
Javier Rodríguez Lagunas* \\ Marco A. Leyva Piña** \\ Juan Manuel Hernández Vázquez ${ }^{* * *}$
}

\section{RESUMEN}

En las universidades mexicanas se han dado cambios de diversa naturaleza para mejorar el perfil de los futuros profesionistas, para acercarlos a las necesidades de su sociedad. Muchos de ellos implican ajustes del currículum escolar, la manera de hacer la docencia, el perfil de los académicos, las formas de gestión universitaria, así como aditamentos innovadores tecnológicamente. Lo que se presentó, buscó establecer los alcances y dificultades de las reformas habidas, considerando las observaciones y perspectivas de los universitarios. También se pretendió establecer algunos parámetros comparativos, debido a los últimos planteamientos de la política educativa que se observan con el nuevo gobierno.

Palabras clave: Reformas universitarias, políticas de educación superior, cambio del currículum escolar, calidad educativa, perspectivas de los universitarios.

\section{ABSTRACT}

In mexican universities, there have been changes of various kinds to improve the profile of future professionals, to bring them closer to the needs of their society. Many of them involve adjustments to the school curriculum, the way of teaching, the profile of academics, the forms of university management, as well as technologically innovative attachments. What is presented seeks to establish the scope and difficulties of the reforms, considering the observations and perspectives of university students. It is also intended to establish some comparative parameters given the latest approaches to education policy that are observed with the new government. Keywords: University reforms, higher education policies, school curriculum change, educational quality, university perspectives.

Recibido 04 de octubre de 2019 y aceptado 29 de octubre de 2019

*Profesor-Investigador de la Universidad Autónoma Metropolitana-Unidad Iztapalapa <rolja56@gmail.com>.

${ }^{* *}$ Profesor-Investigador de la Universidad Autónoma Metropolitana-Unidad Iztapalapa <marcolp2ooo@yahoo.com.mx>.

${ }^{* * *}$ Profesor-Investigador de la Universidad Autónoma Metropolitana-Unidad Iztapalapa <moyml82@gmail.com>. 


\section{INTRODUCCIÓN}

Tras tres décadas de ajustes y reformas para la mayor parte de las instituciones de educación superior (IES), prácticamente todas las universidades públicas del país fueron llevadas a realizar reformas en sus modelos educativos, en sus prácticas docentes, así como en fortalecer el perfil de los académicos y establecer su evaluación (Galaz Fontes y Gil Antón, 2009; Buendía, García, Grediaga, Landesman et al., 20I7), promovido y desarrollado en un contexto y circunstancias del desarrollo del país con signos neoliberales al que de distintas formas de argumentación se avino cualquier proyecto o programa relacionado con la educación superior. Lo más reincidente fue que el país no podía quedar fuera del proceso global al que todas las sociedades están yendo. Desde el discurso del Programa de Modernización de la Educación (Presidencia de la República, 1989), el gobierno señalaba, con esos argumentos, el camino de la modernización de la educación en general y de la superior en particular, dando los trazos principales: renovar el modelo educativo; profesionalizar a los maestros y desarrollar infraestructura ad hoc con base en las nuevas TIC en el campo educativo. El argumento para ello fue calificar de tradicional y caduco el modelo educativo en curso y a los que lo aplicaban. No es este el espacio para establecer y discutir si eran o no tradicionales y caducos los rasgos de semejante modelo y personal docente; lo importante es que desde ese discurso el gobierno federal inició, sin más, el proceso de cambios que fue bien visto por la mirada de los organismos internacionales de economía (sic) y también por la UNESCO, que igualmente emulaba proyectos de renovación para acceder a la sociedad del conocimiento que debía estar en el siglo veintiuno.

Así, los académicos de las IEs públicas, particularmente de las universidades, tuvieron apoyo para fortalecerse en sus estudios específicos sobre la docencia, como también a su perfil disciplinario (PROMEP, hoy PRODEP, pertenencia al sin; en algunas universidades públicas hubo, además, becas al desempeño docente y a la investigación, estímulos a los grados académicos logrados), estancias sabáticas, estancias bibliográficas, así como el acceso a fondos concursables para proyectos académicos, muchos de ellos organizados, aunque no solamente, a partir del CONACyT, todo lo cual fomentó las diferencias en capacidades de desempeño con sus consecuencias en los 
ingresos económicos y en el prestigio (Grediaga Kuri et. al., 2004; Galaz Fontes et al., 2009; Buendía et al., 2017).

En el caso de los alumnos, los ajustes buscaron el acompañamiento más puntual durante sus estudios, con las tutorías académicas ligadas a estímulos económicos especialmente del pRONABEs; los programas de movilidad estudiantil, hasta el cambio curricular de las carreras a nivel licenciatura y la generación de habilidades ligadas a la expresión oral y escrita, a las capacidades de comunicación en idiomas extranjeros, al trabajo en equipo, al compromiso con su propia formación, entre lo destacable (Programas sectoriales de educación 1989; 200I-2006; 2007-20I2).

En medio de estas transformaciones, se entreteje el aspecto propiamente educativo en donde cuestiones de qué formar, para qué formar y cómo formarlo, son las relevantes. Los lineamientos internacionales, en un escenario global, lo definen como educación a lo largo de la vida, se hacen formulaciones pedagógicas y didácticas, en las que se involucra el concepto de calidad educativa, como aspecto toral en la formulación del modelo educativo (UNESCO, I998; BM; 2003), y se advierte de la necesidad de que se formulen vínculos mayores a las necesidades de la economía, de acuerdo con los organismos financieros (Organización para la Cooperación y el Desarrollo [OCDE], 2004a, 2004b). Desde luego, igual que en los otros ambientes, las implementaciones corren toda suerte de ajustes para hacerlos factibles en el nuevo campo. En el educativo, se amalgaman o combinan con nociones, estas sí, propias de su ambiente, provenientes por ejemplo de las vertidas en el conocido Informe Delors (I996) a la UNESCO, pero redireccionadas por los principios de calidad y pertinencia.

Actualmente, con el nuevo gobierno federal se ha dado paso a una suerte de ajustes, que posiblemente vayan en sentido contrario, por lo menos parcialmente, a lo que hasta hoy ha ocurrido en las universidades públicas. Todo lo que podrían ser los logros académicos, al mejorar su perfil y generar en las universidades la figura de la carrera académica, han sido catalogados como neoliberales y su columna vertebral definida como productivista. En la práctica, se han tomado las primeras medidas, bajo el principio de la austeridad republicana -que el propio presidente señala que puede, dado el caso, ser franciscana- de limitar o de plano quitar los apoyos financieros para el pago de los bonos, becas y demás estímulos económicos de los académicos. 
En el caso del modelo educativo, no parece haber aún una crítica sustantiva del nuevo gobierno, aunque se avizora dado el proyecto de formar, en paralelo a las existentes, Ioo nuevas universidades que estarían resolviendo las necesidades de jóvenes de comunidades de bajo perfil socioeconómico. No obstante, si se da paso a la argumentación antineoliberal, pues entonces el modelo educativo, en tanto se estaría orientando por las necesidades del mercado, muy posiblemente sea revalorado, ajustado y eventualmente cambiado, como política de Estado.

Debido a lo anterior, las universidades públicas del país se encuentran, literalmente, entre la espada y la pared, dado el perfil neoliberal de tres décadas pasadas, y el antineoliberalismo que acaba de emerger en el discurso del reciente gobierno federal.

En este contexto, se intenta revalorar la información que se tiene hasta el momento, usando los datos de encuestas realizadas, por un lado, a estudiantes (20I4) y, por otro, a académicos (2015) de cuatro universidades públicas, ambas en torno a las reformas educativas que se han gestado y operado en sus instituciones.

Sugerimos un planteamiento a desarrollar: la participación de las comunidades universitarias en las reformas no fue tan importante como supondría ser parte de una cultura universitaria participativa y propositiva. Además, se aprecia una cierta lejanía de los estudiantes sobre el impacto causado en ellos, no obstante que se considera una opinión general de buen sentido de los cambios; asimismo, los académicos, pese al paso de los años, mantienen cierto escepticismo sobre los logros de las reformas aplicadas e incluso llegan a tomar posturas muy críticas del alcance realmente logrado. Además, en el caso de los académicos, se hicieron un cúmulo importante de observaciones pretendiendo con ello mejorar o incluso corregir lo que se ha hecho.

De todo ello, lo expresado parece ser una advertencia y cuestionamiento a la vez. ¿Cómo deben hacerles las propuestas del cambio en las ies mexicanas para que surtan efectos en favor de las universidades y sus funciones sustantivas? (De Vries y Álvarez, 20I4). Parece no bastar las ideas e intenciones basadas en proyectos internacionales que luego se retoman en los proyectos nacionales, a partir de las políticas públicas abocadas a la educación superior. Hace falta algo más. 


\section{LA PERSPECTIVA DE ANÁLISIS}

El concepto de la reforma llevada al plano de las IEs se inscribe en la reforma del Estado, en constante ajuste dado los objetivos y metas que se ha planteado. No se trata de una reforma tan solo dada por parámetros de corte administrativo, sino del sentido, los fines y valores de las instituciones de educación superior, es decir, una reforma política institucional (Acosta, 2003; Kent, 1999, 2009).

El proceso en su conjunto implica diversas mediaciones como las relaciones de poder y consenso ligadas a las propiamente de conocimiento, en las propuestas de reformas educativas (Popkewitz, 2000); las de las formas de gestión del cambio, amparadas en ejercicios de planeación y organización de las instituciones educativas a partir de la revisión, el análisis y el acuerdo con los partícipes de cambio (Montaño, 20I2), y las de la construcción y reconstrucción de las identidades sociales que constituyen a los sujetos educativos en contextos de cambio de la educación (Touraine, 1994).

En ese sentido, para interpretar las reformas y su capacidad de resolución, es necesario esclarecer la orientación reflexiva de los actores del cambio que no pueden ser concebidos solo como elementos manipulables en el proceso. Desde una perspectiva socioeducativa, los sujetos universitarios son copartícipes del proceso universitario que incluye centralmente la formación profesional. Sus ideas sobre lo que son, pasan por las nociones-fuerza de la institución pública, cual una mediación fundamental de su vida: la responsabilidad social de la universidad, el sentido de equidad social que la permea, los fines universales del saber y el conocimiento, la ética de la profesión, entre lo relevante. Si los cambios comprometen parte de eso, ¿qué perspectiva toman los universitarios?

La perspectiva del sujeto universitario se ofrece, además, dentro de sus circunstancias específicas. Las de ahora están permeadas por la autonomía de las universidades públicas, por el concepto trascendente de la libertad de cátedra, y también se ubican en un entramado de posiciones y acciones de la institución a partir de sus formas de organización y a la manera de gestionar el cambio. En ese conjunto complejo de mediaciones es dable entender o captar la perspectiva del sujeto universitario. Hay eventos, en ese sentido, que los enmarcan y les plantean su escenario; la política educativa 
del Estado es uno de ellos, a la vez que plantea el cambio por mediación de la calidad y la cercanía al mercado, plantea la inclusión social a las universidades como un derecho. Las observaciones de los universitarios que en estas líneas desarrollaremos tienen que ver con su especificidad, y es el de la política educativa que ha impulsado el cambio como lo hemos descrito. No será posible ir más allá, sino como meros apuntes que la situación actual nos narra; no obstante, su descripción analítica y reflexiva nos impone una lógica que trasciende el instante y se ubica en el proceso. Atendiendo a ello, lo que encontramos dibuja una panorámica orientadora de los sujetos ante eventos que, como lo establecimos, ahora están ocurriendo, si bien como meros bocetos, pero con fuerza política.

\section{NOTA METODOLÓGICA}

En nuestro ejercicio de búsqueda de información de los universitarios no podemos examinar por completo, sino de un modo inicial, el sentido colectivo que impera en los estudiantes a partir de observaciones vía la encuesta. En la encuesta nos hemos exigido observar y reconstruir dinámicas (desde luego, instaladas en sus procesos) a partir de cuatro líneas de interpretación: (I) el conocimiento informado de los procesos de ajuste o reforma en la docencia; (2) las formas de participación en dichos procesos, tanto de los formalmente adoptados por la institución, como aquellos inspirados en la propia inquietud e innovación de los actores; (3) opciones de posibilidad en el desarrollo de la docencia y los servicios, y (4) el compromiso social de la institución. Con lo anterior, pretendemos dibujar un panorama descriptivo de los actores del proceso con el afán de contar con una inicial tipificación de su estatuto de actores frente a las reformas que se dan en sus instituciones.

La encuesta de alumnos, por una parte La reforma universitaria desde la perspectiva de los alumnos, 2014 (RUPA 2OI4), fue aplicada presencialmente a muestras estratificadas de estudiantes por asignación Neyman, para hacer estimaciones por sexo y área académica con un error máximo de $5 \%$, sobre una población determinada en cada caso de universidad por estudiantes regulares, con al menos dos años de estancia en la institución o el 50\% de créditos de la carrera y promedio de al menos 7 de calificaciones. Las po- 
blaciones que finalmente se encuestaron quedaron como sigue: UAM, II34; UAN, 396; UATX, 327; UNACH, 355 estudiantes.

En el caso de la encuesta Reforma Universitaria desde la Perspectiva de los Profesores (RUPP, 20I5), durante la prueba del instrumento a los académicos, consistente en alrededor de 40 ítems, los profesores fueron omisos a toda posibilidad de respuesta, lo que llevó a crear un instrumento mucho más ligero que diera, no obstante, la oportunidad de respuesta. Luego, el levantamiento se hizo de dos formas entre las universidades participantes: en el caso de la UAM, consistió en enviar un cuestionario web a los correos electrónicos de todos los miembros del personal académico de la UAM, que a la sazón estaban contratados de manera definitiva. En total, 502 académicos respondieron, los cuales representaron $18.3 \%$ de la plantilla académica definitiva de tiempo completo reportada por la institución. Esta cifra podría parecer baja, pero en realidad no lo es si se considera que la participación fue completamente voluntaria y que se recabaron cerca de 1380 propuestas de mejora, escritas por los académicos de manera abierta y anónima. En el caso de las otras universidades, la UATX, UAN y la UNACH, el levantamiento no se hizo con muestras, sino aplicando a quienes se encontraban en activo, tenían la característica exigida de por lo menos cinco años de antigüedad, y podían responder el cuestionario, tratando de hacerlo de modo presencial, o bien dejando el instrumento para ser respondido y luego entregado (lo que supuso la pérdida de muchos casos). La población finalmente encuestada quedó como sigue: UAM, 502; UAN, 380; UATX, 243, y UNACH, 215 profesores.

\section{EL CAMBIO EN LAS IES, SU SUSTENTO Y COMPLICACIÓN}

Después de más de treinta años de transformación de las bases de la educación superior, dirigido por las orientaciones internacionales de los principales organismos de educación y de economía en el mundo (UNESCO, BM, OCDE), los cambios en las IES ya tienen su lógica e imperan globalmente (BM, 2003; OCDE, UNESCO, 2002; OCDE, 2004; 2008) con sus adecuaciones, limitaciones y problemáticas derivadas: (I) es una educación basada en principios de calidad educativa -con las proclividades hacia el mercado y con una lógica productivista inequívoca-, si bien no hay homogeneidad, ni plataformas uniformes; (2) los propósitos de formación profesional ya operan como los 
nuevos perfiles formadores, muy ligada a la formación por competencias, sin hacer las mediaciones adecuadas entre los saberes disciplinarios y sus prácticas tanto teóricas, metodológicas como profesionales (Irigoyen, Jiménez y Acuña, 20II; Díaz Barriga, 20I4; Rojas Moreno, 20I4); (3) su implementación ocurre dentro de los parámetros que, o bien supervisan o bien evalúan diversas instancias, tanto de orden público como privado (CIEEs, COPAES); (4) la masificación, a la par que expansión, de las IES, ocurre en un proceso que presiona a las públicas y ofrece condiciones mejoradas para las instituciones privadas que han elevado su potencial (De Garay, 2015); (5) en la composición del mercado de trabajo, los perfiles profesionales crecen en su definición, aunque con efectos adversos, de abaratamiento de pagos por sus servicios y mayor presión por nuevos puestos de trabajo, y el desempleo profesional crece (Hernández Laos, Ricardo Solís y Ana Stefanovich, 20I3), y (6) el nuevo perfil de los académicos de las IEs que prolifera con segmentos elitistas y otros que se mantienen o con medianas condiciones o precarizados laboralmente (Grediaga Kuri, Rodríguez y Padilla 2004; Rueda Beltrán, 2012).

En esta tesitura, la vida interna de las IEs, su organización y gestión, su modo de gobernarse, han tenido cambios importantes. Un elemento novedoso se incorpora dando una extrańa combinatoria entre formas de gobierno autoritarias y vida colegiada (Acosta, 20Io). En ese marco, el cambio de las IES ha podido unas veces ser dirigido y orientado con creatividad colegiada, y otras veces ser un producto elaborado a partir de decretos de la autoridad. En ese contexto, también la participación de las comunidades ha explorado opciones y mantenido posiciones críticas ante los cambios.

En la coyuntura, los escenarios para las universidades públicas mexicanas se redefinen fuertemente: en la actual Ley General de Educación se elude, de alguna manera, establecer claramente la autonomía de las universidades, para que sea la propia ley superior la que le dicte sus rumbos; en las leyes secundarias se establece que para acceder a la educación superior no debe mediar necesariamente un examen de admisión, con lo cual, dada una demanda creciente de población de jóvenes que egresan de la educación medio-superior, la matrícula podría crecer a niveles inimaginables e imposibles de manejar por las actuales universidades, lo que no se resolverá con la propuesta del nuevo gobierno de crear Ioo nuevas universidades, que por lo pronto 
se piensa ubicar en zonas deprimidas y alejadas de las zonas urbanas donde suelen estar este tipo de instituciones; debido al proyecto de austeridad actual, que aplica a toda instancia pública, incluidas las universidades, sería previsible una disminución de los financiamientos dirigidos a los esquemas de estímulos económicos a los académicos que, por su actual condición de ingresos desde los denominados salarios-base, podrían sufrir un ajuste que los llevaría, de golpe, a las condiciones de inicios de la década de los ochenta del siglo pasado. Entonces, si las reformas neoliberales llevaron su carga de mercado, las que ahora se inician, incitan toda suerte de incertidumbres entre la comunidad académica de las universidades públicas; el problema educativo se asoma en el horizonte cercano bajo la forma de retrocesos desde una lógica pública de autoridad del Estado, en donde los actores centrales del proceso podrían quedar, de nueva cuenta, al margen de las decisiones.

\section{LOS UNIVERSITARIOS FRENTE A LA REFORMA}

Los universitarios no parecen seguir un patrón definido de acción frente a las reformas; antes bien, se colocan ante ello con base en sus propias necesidades y disyuntivas. Por lo demás, se trató de un proceso de ajustes que fue dándose en el tiempo (tal vez podría medirse en cerca de treinta años), de tal suerte que si bien fue perceptible para muchos, no necesariamente significó un cambio súbito que, de seguro, habría generado respuestas más determinantes. Por ejemplo entre los estudiantes, los ajustes no tocaron la columna vertebral de las licenciaturas, sino hasta entrado al siglo XXI, cuando tuvo significación. Los elementos de corte administrativo y de la gestión de las instituciones (como el tema de la autonomía y de las formas de poder locales), si bien fueron importantes, no provocaron, por ejemplo, entre los estudiantes universitarios observaciones mayores que la evidencia de que se estaban presentando. Lo que sí fue significativo fue aquello que implicaba ajustar, por ejemplo en la UNAM, el ingreso de los estudiantes (el pase automático) y las cuotas de inscripción y colegiatura. Ello sí tuvo una gran reacción tanto que generó el movimiento del CEU expresado de modo contundente en las manifestaciones de 1986 y 1987 (Acuña, 1987). Luego siguió otro movimiento, el del 1999 del CGH, que terminó paralizando a la UNAM prácticamente todo el año por razones similares (Dorantes, 2004). 
Pero en las universidades públicas del país ello no tuvo repercusiones evidentes, de modo que en muchas de ellas hubo ajustes de ambos tipos (ingresos y cuotas) sin que hubiera reacciones determinantes como las de la UNAM (Kent, 1999). Otro ha sido el tipo de repercusiones que fueron causando en la década que está por terminar; por ejemplo, el de los ańos 2012 y 2014 tanto del movimiento \# soy I32, de los chicos Ibero, que así se definieron en el espectro político del cambio de gobierno (Morales Sierra, 20I4), y el de los estudiantes del IPN en contra del nuevo Reglamento Interno y de los planes y programas de estudio (Rivera, 20I4). Todos esos casos podría decirse que fueron políticamente muy relevantes.

En las otras direcciones, la de los ajustes más sinuosos que sin embargo calan igual de hondo, los ajustes del tipo curricular y de las formas pedagógicas, la actitud prevaleciente de los estudiantes podría estar más en el campo de las posibles implementaciones, más que en el de la valoración de su fuerza y posibilidades para su futuro profesional. En cuanto a los académicos, para una parte estable laboralmente implicó su entrada a los cambios vía los sistemas de estímulos y beneficio económicos, y para otros su difícil acceso a las universidades significó precarización salarial e inseguridad laboral (Grediaga Kuri et al., 2004; Bautista et al., 20I7). Pero fueron confluyendo en la conformación de un corpus académico que debía dar respuesta al incremento de la matrícula universitaria y que, ligado al proceso de expansión de las IES mexicanas, expresaba un incremento de número como nunca antes se tuvo conocimiento (Galaz Fontes y Gil Antón, 2009). En última instancia, las experiencias para muchos académicos de universidades públicas los ajustes, en tanto venían acompańados de apoyos financieros, fueron aceptados por sus universidades sin mayor problema (Martínez Lobato, 20I6). No obstante que fuera un proceso complejo que podría llevar a largos aprendizajes y más difíciles implementaciones, las universidades vieron en los ajustes, en particular denominados como flexibilidad curricular, una oportunidad de modernización e innovación sin reparar en la concepción y valoración justamente por los profesores universitarios que tendrían que pasar por ser sujetos activos en ese proceso, lo que implica dotarles de espacios y condiciones tales que, con autonomía y libertad de cátedra, puedan desarrollar sus innovaciones curriculares (Díaz Barriga, 2010). 


\section{LA PERSPECTIVA DEL CAMBIO ENTRE LOS ESTUDIANTES}

Nuestra intención de análisis se atiene a usar solo algunos constructos generales que nos ofrece la información de la encuesta RUPA 20I4, concretados en cinco índices: APD, Adecuación de la práctica docente a la política educativa, desde la perspectiva de los alumnos; OAA, Opiniones sobre la actividad académica; PE, Participación estudiantil en las reformas; Osu, Opiniones sobre los servicios universitarios, y ops, Opiniones sobre el proyecto social de la universidad. El detalle de lo que conforma cada índice se tiene en la tabla Ar del Anexo.

En la tabla I, los valores específicos del índice APD dan cuenta de un nivel de aplicación, por arriba del punto medio, de los cambios según los alumnos, en el ámbito de la docencia universitaria. No parece haber grandes diferencias en las opiniones de los alumnos considerando cada universidad. Entonces, podría suponerse que habría evidencias de esos ajustes. Implica también que los ajustes en los PyPE, sobre todo relacionados con métodos de trabajo pedagógico y didáctico, fueron implementados por los profesores en el aula, lo que lleva a conjeturar que estos tuvieron las habilitaciones mínimas para poder hacerlo.

Tabla I. Media de los indices, por institución. Encuesta rupa 2014

\begin{tabular}{lccccc}
\hline Universidad/ Índice & apd & oaa & ops & osu & pe \\
\hline Min-Max P. medio & IO - 40-25 & II - $44-27.5$ & $6-24-$ I & 0- I4. - 7 & I2 - 48-30 \\
\hline UAM & 31.5 & 34.3 & 20.3 & 8.4 & 23.9 \\
\hline UAN & 30.2 & 37.9 & I9.4 & 7.1 & 23.4 \\
\hline UATX & 30.1 & 36.6 & I9.6 & 6.1 & 23.0 \\
\hline UNACH & 29.7 & 32.8 & I9.I & 6.I & 23.5 \\
\hline
\end{tabular}

Fuente: Cálculos propios, con base en la encuesta rupa (La reforma universitaria desde la perspectiva de los alumnos, 2014). (APD), Adecuación de la Práctica Docente a la política educativa, desde la perspectiva de los alumnos; (PE), Participación Estudiantil en las Reformas; (OAA), 
Opiniones sobre la actividad académica; (OSU), Opiniones sobre los servicios universitarios; (OPS), Opiniones sobre el proyecto social de la universidad.

Lo anterior va de la mano con los resultados encontrados en el índice OAA, pues su construcción atañe a indicadores que denotan la capacidad personal del alumno para abrirse paso no solo en sus estudios, sino mirarse en perspectiva como poseedor de conocimientos científicos, humanistas, técnicos, con los cuales incorporarse al mundo como profesionista e incluso hacer estudios de posgrado. En este caso, el índice siempre se coloca entre 5 y Io puntos por arriba de la media de la escala en las opiniones de los estudiantes considerando, ahora sí, las diferencias entre universidades. Obsérvese que la brecha entre las universidades extremas ronda los 5 puntos.

Ligado a ello, se tiene lo que expresa el índica ops que implica a un factor fundamental de la universidad pública, su compromiso con la sociedad. Los valores obtenidos (tabla I) siempre van por arriba del punto medio de la escala, cerca del 20, para todos los casos. Evidentemente, los alumnos sí consideran que se incorporan en su formación los valores éticos, la conciencia y valores sociales, así como la discusión de los problemas nacionales. A nuestro entender, ello es parte de la idea de universidad pública de la que hay ya un imaginario social que no se puede eludir.

En cuanto a los resultados del índice PE, lo evidente es que los valores encontrados van por abajo del punto medio de la escala, que es de 30 . Este índice se compone de indicadores que grosso modo podemos ubicar en dos ámbitos: las iniciativas de los alumnos por desarrollar actividades de desarrollo académico fuera de las clases, incorporando aditamentos tecnológicos como el internet, asistir a eventos como las presentaciones de libros, tomar el libro más allá de lo que los cursos le exigen; por otro lado, la manera en que atienden la información sobre las reformas en su institución y su capacidad de involucramiento en ello a través de diversas instancias, incluyendo las colegiadas.

Los resultados del índice PE, por lo menos en lo tocante a su capacidad de involucramiento, desnudan ese resabio de la cultura universitaria que se niega a reconocerle posiciones activas al alumnado a la hora de decidir los rumbos de la universidad. Es decir, partimos de la observación de que esta forma de no participar en lo tocante a la reforma de su institución, como en 
otros aspectos de la vida universitaria, no pueden simplemente ser referidas a la conciencia y voluntad personales, o grupales, de los alumnos, sino que implica a una cultura de las instituciones educativas que les ha generado esa condición que, ahora, se refuerza por la propia cultura de estas generaciones de jóvenes mexicanos, en donde hay tantas cuestiones de la vida masificada y consumista que atender, que termina por perderse de foco la reflexión de su ser universitario y lo que conlleva. Esto cabe tanto para la UAM, donde posiblemente se hayan construido ya espacios más amplios de cultura estudiantil participativa, como para la UAN, UNACH y UATX.

\section{LA PERSPECTIVA DE LOS ACADÉMICOS ANTE LAS REFORMAS}

La idea básica que se ha manejado es incorporarlos a dinámicas de constante superación académica, con el afán de conformar un cuerpo social de alto nivel que establezca las bases de la transformación de la universidad hacia la calidad educativa. En ello tiene cuenta tanto el SNI, el PRODEP y los sistemas de estímulos que en cada institución se han podido dar. No obstante que en cada universidad las incorporaciones tienen sus altibajos, de orden presupuestal y de gestión, se puede decir que los ajustes gradualmente constituyeron una academia que, sin embargo, se desdobla en varios tipos: académicos que acceden a todos los sistemas de estímulos y se incorpora al SNI; académicos que siendo contratados de manera definitiva no han podido, o querido, entrar en la dinámica del productivismo académico, y finalmente académicos que acompañan a ambos en las universidades, pero son contratados temporalmente, por horas o de tiempo parcial y por ello no acceden a ningún apoyo como los referidos, no entran en el productivismo, sino en la precariedad laboral. El cuadro final parece un corpus segmentado antes que otro idealmente integrado (Grediaga Kuri et al., 2004; Galaz y Gil, 2009; Gil, 2018).

En los casos de los académicos de las universidades analizadas, de acuerdo con la tabla 2, los datos refuerzan la idea de un corpus académico segmentado. 
Javier Rodríguez, Marco A. Leyva y Juan Hernández

Tabla 2. Rasgos del Personal académico en la uam, uan, uatx y unach (2015) (\%)

\begin{tabular}{lccccc}
\hline Universidad & $\begin{array}{c}\text { Definitivos } \\
\text { tc (\% del } \\
\text { total) }\end{array}$ & $\begin{array}{c}\text { Doctores } \\
\text { (\% del } \\
\text { total) }\end{array}$ & $\begin{array}{c}\text { Becas, estímulos } \\
\text { económicos } \\
\text { (\% del total) }\end{array}$ & $\begin{array}{c}\text { PRODEP } \\
\text { (\% del } \\
\text { total) }\end{array}$ & $\begin{array}{c}\text { (\% de } \\
\text { tc) }\end{array}$ \\
\hline uam & 89 & 56 & 75 & $4 \mathrm{I}$ & 40 \\
\hline UAN & 85 & 24 & --- & 40 & IO \\
\hline uatx & 43 & 34 & --- & --- & II \\
\hline unach & 43 & 30 & --- & I3 & 4 \\
\hline
\end{tabular}

Fuente: uam, uan, uatx y unach, informes oficiales de las universidades.

Este perfil de la academia de estas universidades nos revela el real y también limitado potencial habido para contender con los procesos de reforma en que se vieron envueltos y que fueron planeados desde una lógica productivista que la política pública ha manejado en décadas.

De los casos analizados, podemos decir que la UAM tiene altos niveles de ajuste al modelo de productividad, mientras que en las otras tres universidades aparecen actores y dinámicas que obstruyen dicho acceso tanto en el sentido de las capacidades de gestión e interlocución de sus directivas con agentes gubernamentales y estatales, como de las dificultades intrínsecas de las universidades para planificar y organizar su desarrollo (Orozco et al., 200I5; Castañeda et al., 2015; Mecalco et al., 2015).

Uno de los resultados de la investigación es que la participación en el sistema de becas y estímulos tiene efectos dispares; por una parte, los profesores consideran en un alto porcentaje, siempre mayor al 70\% de los casos, que este sistema tiende a mejorar sus funciones docentes (tabla 3), pero, en las entrevistas, también reconocen que hay desigualdades en las posibilidades de acceso y que se genera un sistema de diferenciaciones sociales en el interior de sus universidades. Huelga decir que en tres de las cuatro universidades valoradas, tales sistemas de estímulos se encuentran poco o nada desarrollados; por tanto, podría ser visto como algo que se busca, pero no se tiene.

Un asunto medular es que la encuesta reporta que no hay un convencimiento de que los ajustes sean adecuados o efectivos tanto respecto a los PPE tanto a su perfil formador, como en la idea de éxito profesional, ni de que una parte medular de esos planes de estudio, como es la interdisciplina, sea 
considerada un aspecto importante para la formación profesional, lo mismo que las tutorías (detalles en tabla 3).

En cambio, la habilitación de la docencia, de acuerdo con los datos mostrados en la tabla 4, implica que debe fortalecerse, lo que tiene varios significados, uno de los cuales es que hay una actitud autocrítica de los académicos sobre su propia habilitación para el trabajo docente.

Algo que llama la atención de los resultados mostrados en la tabla 3 es que la academia está haciendo un esfuerzo por consolidarse en su medio a partir de la vinculación universitaria entre pares, si bien hay diferencias entre instituciones (detalles en tabla 3 ).

Tabla 3. Porcentaje (\%) de acuerdo según aspecto de la actividad y universidad, 2015

\begin{tabular}{|c|c|c|c|c|}
\hline Aspecto de la actividad académica & UAM & UAN & UATX & UNACH \\
\hline $\begin{array}{l}\text { Mi universidad apoya a los docentes para realizar } \\
\text { estudios de posgrado }\end{array}$ & $83 . \mathrm{I}$ & 78 & $8 \mathrm{I}$ & 80 \\
\hline $\begin{array}{l}\text { Necesito tomar cursos de didáctica para mejorar mi } \\
\text { práctica docente }\end{array}$ & 6I.8 & 87 & 86 & $77 \cdot 7$ \\
\hline $\begin{array}{l}\text { Las becas y estímulos fomentan la mejora de mi } \\
\text { actividad docente }\end{array}$ & 70.3 & 70 & 70 & $75 \cdot 3$ \\
\hline $\begin{array}{l}\text { El plan de estudios vigente favorece una inserción } \\
\text { laboral exitosa en los estudiantes }\end{array}$ & 53.0 & 69 & - & 56.9 \\
\hline $\begin{array}{l}\text { Las UEAS que los alumnos toman de otras carreras } \\
\text { como optativas contribuyen a mejorar su formación }\end{array}$ & 67.1 & 46 & - & 55 \\
\hline $\begin{array}{l}\text { El perfil de egresos del plan de estudios responde a } \\
\text { lo que la sociedad requiere }\end{array}$ & $57 \cdot 2$ & 75 & -- & 62.7 \\
\hline $\begin{array}{l}\text { En mi universidad los docentes participan en la for- } \\
\text { mación académica del estudiante }\end{array}$ & $74 \cdot 3$ & $75^{*}$ & $72^{*}$ & 60 \\
\hline $\begin{array}{l}\text { El programa de tutorías contribuye en la formación } \\
\text { académica del estudiante }\end{array}$ & $5 \mathrm{I} .8$ & 67 & 72 & $57 \cdot 3$ \\
\hline $\begin{array}{l}\text { El programa de tutorías contribuye a la formación } \\
\text { personal del estudiante }\end{array}$ & 51.6 & 60 & 72 & -- \\
\hline Participo en redes de colaboración académica & 72.3 & 74 & - & $57 \cdot 3$ \\
\hline $\begin{array}{l}\text { Frecuentemente busco información sobre los proce- } \\
\text { sos de reforma universitaria }\end{array}$ & $57 \cdot 4$ & 68 & 73 & 61.5 \\
\hline $\begin{array}{l}\text { Mi universidad y mi carrera necesitan más cambios } \\
\text { que los logrados hasta ahora }\end{array}$ & 87.5 & 90 & 90 & 46.9 \\
\hline
\end{tabular}

Fuente: Cálculos propios, con base en la encuesta ruppi, 20I5.

${ }^{*}$ En mi universidad los docentes participan en la construcción de propuestas académicas. 
Un espacio de confluencia mayor de los académicos, casi del 90\% de las opiniones, salvo en la UNACH, es respecto a la necesidad de hacer mejoras a lo que hasta ahora se tiene de reformas aplicadas. Es decir, o lo generado no es suficiente, o lo que se ha hecho no es adecuado.

Tabla 4. Distribución porcentual (\%) de las propuestas de los académicos, según temática y universidad, encuesta RUPPI 2015

\begin{tabular}{|c|c|c|c|}
\hline \multirow{2}{*}{$\begin{array}{l}\text { Temática de las propuestas } \\
\text { Gestión académica }\end{array}$} & UAM & UAN & UATX \\
\hline & 30.0 & & 15.0 \\
\hline Enseñanza-aprendizaje & 20.2 & 22.6 & 22.7 \\
\hline Planes y programas de estudio & I8.4 & & \\
\hline Vinculación & 15.0 & 6.8 & 9.18 \\
\hline Gestión en infraestructura y equipamiento & 7.0 & & \\
\hline Gestión administrativa & $3 . \mathrm{I}$ & 30.5 & $16.6^{*}$ \\
\hline Gestión en servicios de apoyo & 2.8 & & \\
\hline Gestión en normatividad & $\mathrm{I} .4$ & & \\
\hline Aplicar Reforma & & II.6 & \\
\hline Función social & & I.4 & \\
\hline Investigación & & 3.9 & I.3 \\
\hline Otras & 2.2 & I2.9 & 15.2 \\
\hline No tiene opinión & & I0.3 & 20.0 \\
\hline Total & IOO.O & IOO.O & IOO.0 \\
\hline
\end{tabular}

Fuente: Informes técnicos del Proyecto "Los universitarios frente al cambio. Reforma educativa $y$ proyecto social. Una perspectiva regional" Fondo SEP-CONACYT, 2018.

*Incluyen en este rubro: carga académica, mejoras laborales, infraestructura, estudiantes y gestión $y$ normatividad.

Las propuestas vistas en forma agregada muestran los cambios necesarios más allá de los aplicados por sus autoridades: (I) mejoramiento de la gestión docente, ligado a la implementación de infraestructura y servicios adecuados; (2) mejoramiento de la gestión administrativa y manejo adecuado de la normatividad, y (3) la vinculación universitaria. Lo que parece dirigir la brújula hacia la capacidad de gestión en las universidades consideradas y, desde luego, involucra a la profesionalización de sus direcciones. 


\section{A MANERA DE CIERRE}

Los procesos de ajuste, neoliberalmente concebidos y desarrollados en las universidades mexicanas, y que podemos valorar en la exploración de las cuatro universidades analizadas, tienen su concreción en unas más que en otras. Diríase que en las estatales el problema es remontar dificultades mayores que en las federales tipo UAM: escasez de recursos, falta de probidad en los cargos y manejos del financiamiento, escaso desarrollo de la carrera académica por la poca atracción que les pueden significar a perfiles de académicos bien desarrollados, pero que de entrar serían relativamente mal pagados. La academia, como quiera que sea, ha intentado incursionar en los nuevos modelos educativos y sus implementaciones, aunque las más de las veces con raquíticos insumos e infraestructuras para lograrlo. No obstante que en uno de los casos la UAM, se tiene la información de que ha logrado atraer suficientes recursos tanto del presupuesto regular como de los programas extraordinarios para involucrarse en mayor medida a los cambios. Ese no ha sido el caso de las otras universidades valoradas. Ello compromete una revisión mayor de lo encontrado que, no obstante, podría llevarnos a establecer que los ajustes en los sistemas de educación superior son un asunto de mayor relevancia y no solo de contar con recursos financieros y aditamentos básicos. En realidad, cuando se trata de innovar educativamente se puede pensar en poner en marcha una gran dinámica institucional que invoque a su academia a incorporarse de un modo no solo consciente de sus implicaciones, sino reflexivo de lo que implica. Es una tarea titánica (Díaz Barriga, 20IO).

Los estudiantes conforman un corpus social diverso no solo socioeconómico, sino cultural. El acceso a determinados bienes materiales y culturales puede limitarlos o dotarlos de mayores oportunidades en sus estudios. En su perspectiva, los cambios planeados ocurren, pero no necesariamente pueden manejarlos con autonomía, posiblemente porque hay segmentos de ellos que adolecen de cultura del estudio y condiciones para lograrlo. Su lógica como estudiante los aleja, a muchos, de incidir en las dinámicas universitarias relativas a ser agentes propositivos del currículum escolar y de las pedagogías ad hoc con los nuevos tiempos. Sus dificultades sobrepasan a sus posibilidades y, aún, la política educativa no habría podido verse coronada con éxito para 
contar con profesionistas portadores de las nuevas habilidades y capacidades que se están sugiriendo más allá del conocimiento disciplinario. En ello, al parecer, no hay graves diferencias de concepto entre las universidades consideradas: unas tendrán más posibilidades de recursos que otras, pero en ambos tipos no parece incorporarse una concepción más decantada del ajuste educativo que conlleve una interiorización de su concepto. Es decir, pasar de una enseñanza calificada de rígida a otra de tipo flexible, implica un conjunto de mediaciones relativas al concepto de aprendizaje, al concepto de comunicación dialógica, a los procesos vinculatorios entre profesión y trabajo profesional, entre otros, que es necesario precisar, repensar, aclarar para luego poder resolver no discursiva, sino prácticamente en el proceso formativo superior.

Ante un panorama podríamos decir de proceso inacabado, no obstante todo lo hecho en las universidades públicas mexicanas, que ya solo con los ejemplos nos parece mostrar un mapa de diversidad y de desigualdades, la transición a que ahora se le está llevando, marcada por el incremento mayor de su matrícula (y con la pretensión de liberar los accesos de todo filtro incluyendo los exámenes de admisión), que pone en jaque las capacidades de las instituciones, no solo en cuanto a infraestructuras, que ya es un gran problema, sino al inacabado y segmentado proceso de conformar una academia robusta, podría llevar a desbalances inmediatos que darían pie a nuevas inestabilidades de las IEs públicas mexicanas, ahora ante la pretensión de lograr una enseńanza de excelencia donde la calidad parece contar menos o incluso desaparecer del escenario.

\section{REFERENCIAS}

Acosta, A. (2003). Estado, politicas y universidades en un periodo de transición. Universidad de Guadalajara, Centro Universitario, FCE. Acosta, A. (2010). Príncipes, burócratas y gerentes. El gobierno de las universidades públicas en México. México: Biblioteca de la Educación Superior, ANUIES, UUAL.

Acuña, A. (1987). Cronología del movimiento estudiantil de 1986-1987. Cuadernos Politicos, 49(50), 86-96. <https://ses.unam.mx/ 
curso2018/materiales/Acunar987_CronologiaDelMovimiento Estudiantil.pdf $>$.

Banco Mundial (2003). Aprendizaje permanente en la economía global del conocimiento: desafios para los paises en desarrollo. Informe del Banco Mundial. Banco Mundial, Alfaomega. <http://siteresources.worldbank.org/EDUCATION/Resourc es/278200-IO99079877269/547664-IO99079984605/LLL_KE_ Spanish.pdf $>$.

Banco Mundial (2003). Construir sociedades del conocimiento: nuevos desafíos para la educación terciaria. Banco Internacional de Reconstrucción y Fomento, Banco Mundial. Washington. <http:// www.bancomundial.org.mx/bancomundial/SitioBM.nsf/>.

Buendía, A., García, S., Grediaga, R., Landesman, M., Rodríguez, R. et al. (2017). Queríamos evaluar y terminamos contando: alternativas para la evaluación del trabajo académico. Sociológica, 32 (92), 309-326. <https://www.redalyc.org/pdf/3050/30505277IOII.pdf>.

Castañeda, Y., Anaya, A., López, J. y Velasco, M. (2018). Las reformas universitarias: entre las prácticas tradicionales y los procesos de mejora en la UNACH. J. En Rodríguez Lagunas y J. Manuel Hernández Vázquez (20I8), Los estudiantes mexicanos ante las reformas universitarias. Un estudio comparado (26I-3IO). UAMI, Ediciones del Lirio.

De Garay, A. (2015). Las instituciones de educación superior privada. Un tema de agenda. El caso de México. XIII Congreso Nacional de Investigación Educativa. Chihuahua, México.

Delors, J. (1996). La educación encierra un tesoro. Informe a la UNESCO de la Comisión Internacional sobre la Educación para el Siglo XXI. Editorial Santillana, unEsCo.

De Vries, W. y Álvarez, G. (20I4). El éxito y el fracaso de las políticas para la educación superior. En H. Muñoz, G. (coord.), La universidad pública en México. Análisis, reflexiones y perspectivas (I5-35). Editorial unam, Seminario de Educación Superior, M. A. Porrúa.

Díaz, Á. (20I4). Construcción de programas de estudio en la perspectiva del enfoque de desarrollo de competencias, Perfiles Educativos, XXXVI (I43), I42-I62. 
Dorantes, L. (2004). La opinión de los estudiantes en el conflicto de 1999 por las reformas al Reglamento General de Pagos de la UnAM. Revista Mexicana de Ciencias Políticas y Sociales, XLVII(19I), I49I95. <https://www.redalyc.org/pdf/42I/42II9IO9.pdf>.

Galaz, J. y Gil, M. (2009). La profesión académica en México: un oficio en proceso de reconfiguración. Revista Electrónica de Investigación Educativa, II(2), I-3I.

Gil, M. (29 de julio de 20I8). Académicos adscritos al SNI, los más consentidos en la enseńanza superior. La Jornada. <https://www. jornada.com.mx/20I8/o7/29/politica/or2nipol>.

Grediaga, R., Rodríguez, J. y Padilla, L. (2004). Políticas públicas y cambios en la profesión académica en México en la última década. Biblioteca de la Educación Superior. ANUies, uam.

Hernández, E., Solís, R. y Stefanovich, A. (Coords.) (2013). Mercado laboral de profesionistas en México. ANUIES.

Irigoyen, J., Jiménez, M. y Acuña, K. (20II). Competencias y educación superior. Revista Mexicana de Investigación Educativa, I6(48), 243-266. <http://www.redalyc.org/articulo.oa?id=I40I556IOII>.

Kent, R. (1999). Cambios emergentes en las universidades públicas ante la modernización de la educación superior. En H. Casanova y R. Rodríguez (coords.), Universidad contemporánea: politica y gobierno. Tomo II (233-254). CeSU, UnAM, Porrúa.

Kent, R. (coord., 2009). Las políticas de educación superior en México durante la modernización. Un análisis regional, ANUIEs. Biblioteca de la Educación Superior.

López, R. (1996). El financiamiento a la educación superior: 1982-1994 (Tesis de maestría, PEstyc, IPN). <file://C:/Users/uami/ Downloads/1996\%2OROMUALDO\%2OLOPEZ\%2OZARATE.pdf $>$. Martínez, L. (20I6). El currículo de la universidad pública mexicana a 20 años de permanecer en los programas de financiamiento extraordinario, Universia, viI(I9). <file://C:/Users/uami/ Desktop/Mart\%C3\%ADnez_Lobatos_ReformaCurricular_ Financiamiento_Educ_Sup2or6.pdf >.

Mecalco, M., Padilla, B., Elizalde, R. y Salazar, E. (2018). Los estudiantes frente a la reforma de la Universidad Autónoma de Tlaxcala (UATX): 
su percepción del cambio. L. J. Rodríguez y V. J. M. Hernández, Los estudiantes mexicanos ante las reformas universitarias. Un estudio comparado (I75-256). CSH-UAMI, Ediciones del Lirio.

Mendoza, J. (20I7). Financiamiento de la educación en la primera mitad del gobierno de Enrique Peńa Nieto: ¿̨fin del periodo de expansión? Perfiles Educativos, XXXIX (I56), II9-I40. <http://www. redalyc.org/pdf/I32/I3250923008.pdf $>$.

Montaño, L. (2012). La organización universitaria. Disputa y tensiones entre el modelo administrativo y la anarquía organizada. En A. Barba Álvarez y O. Lobato Calleros (coords.), Instituciones de educación superior, politicas públicas y organización. Colección Las Ciencias Sociales. UAmI, Porrúa.

Morales, F. (20I4). El movimiento estudiantil \# YoSoyı32. Antología hemerográfica (tesis de maestría en Historia, Universidad Iberoamericana). <http://www.bib.uia.mx/tesis/pdf/or5937/or5937.pdf>. Organización de las Naciones Unidas para la Educación, la Ciencia y la Cultura (unesco, 1998). Conferencia Mundial sobre Educación Superior. La educación superior en el siglo XXI: Visión y acción. París: UNESCO. <http://www.unesco.org/education/educprog/ wche/declaration_spa.htm >.

OCDE, UNESCO (2002). Financing Education-Investments and Returns. Analysis of the World Education Indicators. OCDE, UNESCO.

OCDE (2004a). Internationalization and Trade in Higher Education: Opportunities and Challenges. OCDE.

OCDE (2004b). On the Edge. Securing a Sustainable future for Higher Education (OECD Education Working Papers), 7. OECD.

OCDE (2008). Tertiary Education for the Knowledge Society: OECD Thematic Review of Tertiary Education: Synthesis Report. OCDE.

Orozco, A., Heredia, E. y Pacheco, J. (20I8). Los estudiantes universitarios ante las reformas de la universidad autónoma de Nayarit (UAN). En L. J. Rodríguez y V. J. M. Hernández (coords.), Los estudiantes mexicanos ante las reformas universitarias. Un estudio comparado (99-I72). CSH-UAMI, Ediciones del Lirio.

Popkewitz, T. (2000). Sociología politica de las reformas educativas. Madrid: Morata. 
Presidencia de la República (1989). Programa de Modernización de la Educación. México.

Secretaría de Educación Pública (2007). Programa Sectorial de Educación 2007-20I2. CDMX, México: SEP.

Rivera, G. (20I4). Ejecentral. CDMx, México. <http://www.ejecentral. com.mx/cronologia-de-la-lucha-de-los-estudiantes-del-ipn/>.

Rojas, I. (2OI4). La educación basada en normas de competencia (EBNC) como un nuevo modelo de formación profesional en México. En Á. Valle Flores (coord.), Formación en competencias y certificación profesional. IISUE, UNAM.

Rueda, M. y De Diego, M. (2012). Las políticas de evaluación de los académicos universitarios. Perfiles Educativos, XXXIV (número especial), 93-I06.

Touraine, A. (1994). Critica de la modernidad. FCE.

Universidad Autónoma de Chiapas (2010). Histórico estadístico I9992008. <http://www.planeacion.unach.mx/images/4Estadisticas/ SeriesEstadisticos/sE_99-o8.pdf>.

Universidad Autónoma Metropolitana (2012). Anuario estadístico 2012. México: UAM. <http://www.transparencia.uam.mx/inforganos/ anuarios/anuario20I2/Anuario_Estadistico_20I2.pdf>.

Universidad Autónoma Metropolitana (2016). Anuario estadístico 2016. México: UAM. <http://www.transparencia.uam.mx/inforganos/ anuarios/anuario20I6/anuario_estadistico_20I6.pdf>.

Universidad Autónoma de Nayarit (2016). Informes de labores. Varios años. UAN.

Universidad Autónoma de Tlaxcala (2015). Compendio estadistico 20I020I5. Diseño y análisis estadistico. UAT.

Universidad Autónoma de Tlaxcala (20I6). $2^{\circ}$ informe de actividades. Gestión 20I5-20I6. UAT.

Unesco (1998). Declaración Mundial sobre la Educación Superior en el Siglo XXI: Visión y Acción. <http://www.unesco.org/education/ educprog/wche/declaration_spa.htm $>$. 
Tabla AI. Variables incluidas en la construcción de Índices basados en la encuesta RUPA-UAM 2014

\begin{tabular}{lll}
\hline Índice & Vscala \\
Alpha ordinal кмо & Varibles & Min-Max \\
& & Punto medio \\
\hline
\end{tabular}

I3 Los profesores crean estrategias de aprendizaje que toman en cuenta mis conocimientos previos. $\mathrm{I}_{4}$ Los profesores toman en cuenta las opiniones de los alumnos para trabajar en clase.

Is Los profesores expresan comentarios orales o escritos a los trabajos que realizan los estudiantes.

APD I6 Los profesores estimulan la participación de sus Adecuación de la práctica docente a la política educativa, desde la perspectiva de los alumnos alumnos en clase.

I7 Los profesores estimulan que busque información por mi cuenta para responder mis dudas académicas.

I8 Los profesores escuchan nuestras opiniones aun cuando no estén de acuerdo con ellas.
0.8866
0.8782

Iro Los profesores recurren a debates, ejercicios prácticos o estudios de caso para desarrollar sus clases.

IIs Es común que mis profesores se comuniquen conmigo para fines académicos por Facebook, correo electrónico u otros medios digitales.

II7 Los profesores aprovechan los resultados de los exámenes para revisar temas en que los alumnos salimos mal.

II7 Los profesores creen que lo saben todo.

IIIII Leo más de lo que requieren los programas de los cursos.

PE

Participación estudiantil en las reformas

0.9159

0.8957
IIII3 Comparto información académica en internet.

IIII6 Asisto por iniciativa propia a eventos académicos dentro de la universidad.

IIII8 Asisto a eventos artísticos (cine, teatro, dan- 30 za, etc.) que organiza la universidad.

III22 Solicito que se me asigne un tutor cuando no lo tengo.

III28 Busco información por mi cuenta sobre las reformas universitarias. 


\begin{tabular}{|c|c|}
\hline & $\begin{array}{l}\text { III29 Platico con mis compañeros acerca de las } \\
\text { reformas universitarias. } \\
\text { III30 Participo en alguna organización estudiantil } \\
\text { independiente de las autoridades de la universidad } \\
\text { para promover cambios en mi universidad. } \\
\text { III3I Asisto a las discusiones de los órganos uni- } \\
\text { versitarios para discutir las reformas universitarias. } \\
\text { III32 Participo en discusiones de los órganos uni- } \\
\text { versitarios sobre las reformas universitarias. } \\
\text { III33 Participo en alguna organización externa a la } \\
\text { universidad para promover cambios en ella. } \\
\text { VI4 Siempre que puedo, participo en actividades } \\
\text { que tienden a mejorar los servicios para los uni- } \\
\text { versitarios en la institución }\end{array}$ \\
\hline $\begin{array}{l}\text { OAA } \\
\text { Opiniones sobre la } \\
\text { actividad académica } \\
0.8734 \\
0.8822\end{array}$ & $\begin{array}{l}\text { II23 El programa de tutorías ha contribuido al me- } \\
\text { joramiento de mi desempeño académico. } \\
\text { II24 Mi formación en la universidad me brinda } \\
\text { una sólida base científica. } \\
\text { II25 Mi formación en la universidad me brinda } \\
\text { una sólida base humanística. } \\
\text { II26 Mi formación en la universidad me brinda } \\
\text { una sólida base técnica. } \\
\text { II27 Mi formación en la universidad me acerca al } \\
\text { ejercicio profesional. } \\
\text { II28 Estoy aprendiendo cosas útiles para mi vida } \\
\text { laboral. } \\
\text { II30 Estoy desarrollando habilidades para buscary } \\
\text { organizar información obtenida de internet, bases } \\
\text { de datos u otras fuentes. } \\
\text { II3I Mi formación en la universidad me capacita } \\
\text { para ingresar a un posgrado si así lo decido. } \\
\text { II33 Estoy desarrollando habilidades para aprender } \\
\text { por mi propia cuenta. } \\
\text { Vi7 La vinculación que la institución ha logrado } \\
\text { con el entorno económico de la región es muy } \\
\text { importante. } \\
\text { Vi8 El programa de movilidad de alumnos que } \\
\text { maneja la universidad nos ha dado nuevas opcio- } \\
\text { nes en nuestro desarrollo }\end{array}$ \\
\hline
\end{tabular}


I 33 ¿Tienes una opinión favorable sobre la limpieza en las áreas comunes de la universidad?

I35 ¿Tienes una opinión favorable sobre los servicios del comedor universitario?

I38 ¿Consideras adecuado el mobiliario para el tipo de clases que recibes?

IV8 La red inalámbrica de mi universidad es:

OsU

$\mathrm{V}_{3}$ ¿Cómo calificas los servicios de vigilancia de tu

Opiniones sobre los servicios universitarios universidad? (Tlax).

V8 La forma de realizar los trámites escolares es eficaz.

$\mathrm{V}_{9}$ Los servicios de biblioteca son eficaces.

0.8615

Vıo Los servicios de cómputo son eficaces.

0.8014

VII Los servicios médicos para los alumnos son eficaces.

VI2 Los servicios psicológicos para los alumnos son eficaces.

VI3 Los servicios deportivos para los alumnos son eficaces.

VI5 Las actividades de extensión y difusión de la cultura que organiza la universidad son atractivas. Vig La bolsa de trabajo que maneja la universidad es útil para vincularnos al medio laboral.

Irg En mi carrera se fomenta la ética profesional.

OPS

I20 En mi carrera se fomenta la conciencia de res-

Opiniones sobre el proyecto social de la universidad ponsabilidad social.

I2I En mi carrera se discuten problemas nacionales.

I22 En mi carrera se fomentan los valores sociales. II2I Mi universidad me ayuda a realizarme como

0.8765

0.7802 persona.

II 22 Cuando comento noticias o problemas del país con mi familia, lo hago pensando en lo que he aprendido en mi carrera.

KMO: Índice Kaiser-Meyer-Olkin de adecuación de la muestra.

Nota: La prueba SK de normalidad realizada en STATA, concluyó en que ninguno de los indices se ajusta a la normal, ya que el Pvalor (o.oooo) en la prueba del sesgo resultó en todos los casos inferior a 0.05 .

Fuente: Cálculos propios en STATA, con base en la encuesta La reforma universitaria desde la perspectiva de los alumnos, UAM 2014 